\title{
Refinement of Kripke Models for Dynamics
}

\author{
Francien Dechesne ${ }^{1}$, Simona Orzan ${ }^{1}$, and Yanjing Wang $^{2}$ \\ ${ }^{1}$ Department of Computer Science, Eindhoven University of Technology, \\ P.O. Box 513, NL-5600MB, Eindhoven, The Netherlands \\ ${ }^{2}$ Centrum voor Wiskunde en Informatica, \\ P.O. Box 94079, NL-1090 GB Amsterdam, The Netherlands
}

\begin{abstract}
We propose a property-preserving refinement/abstraction theory for Kripke Modal Labelled Transition Systems incorporating not only state mapping but also label and proposition lumping, in order to have a compact but informative abstraction. We develop a 3-valued version of Public Announcement Logic (PAL) which has a dynamic operator that changes the model in the spirit of public broadcasting. We prove that the refinement relation on static models assures us to safely reason about any dynamic properties in terms of PAL-formulas on the abstraction of a model. The theory is in particular interesting and applicable for an epistemic setting as the example of the Muddy Children puzzle shows, especially in the view of the growing interest for epistemic modelling and (automatic) verification of communication protocols.
\end{abstract}

\section{Introduction}

Epistemic logics are modal logics for reasoning about knowledge, traditionally used to describe the distribution of information among parties. Recently, these logics have become interesting also from a more practical perspective, i.e. for modelling knowledge development during communication protocols, by the addition of dynamics: mathematical constructions that enable to reason about knowledge and information change 812]. Methods based on epistemic logics have been developed for the analysis of complex communication protocols: e.g. BAN logic [4, the theory of function views [13] and interpreted systems 8,1019]. These approaches are also more and more tool-supported, and interesting protocol properties are assessed or discarded by (automatic) model checking [111922].

The structures on which epistemic formulas can be evaluated are Kripke models as in usual modal logic, with multiply labelled transitions representing different agents' uncertainties. Inevitably, when epistemic modelling is applied to complex situations, very large epistemic models can be expected. One way to deal with this, is to import the refinement and abstraction techniques developed for labelled transition systems (LTS), e.g. 16 15 20]. The refinement method intuitively relates a detailed model (refined model) with a coarser one (abstract model) in which some information may be lost, but the information kept is faithful to the detailed model. In the Kripke models of the epistemic setting, there are often transitions with different labels that might be similar to each other — for instance if they express 
uncertainties of agents playing similar roles in a multi-agent system. Another specific characteristic of epistemic Kripke models is that in modelling practical situations, numerous different basic propositions might be used. We may expect to lump some of those transitions with different labels or combine states with different propositional valuations to obtain a more compact abstraction. However, the traditional LTS abstraction techniques do not perform this type of reductions, so an adaptation is needed. Moreover, when we include the dynamic modalities, which essentially change the model, into the language (e.g announcements or actions, cf. 8 1 12 7]), it is a challenge to adapt the LTS abstraction theory such that a suitable abstraction relation will preserve the truth values of the dynamic formulas on the abstract model.

In this paper, we extend the refinement theory for Kripke Modal Labelled Transition Systems (KMLTSs), incorporating not only state mapping but also label- and proposition lumping, in order to obtain compact but informative abstractions. We develop a 3-valued Public Announcement Logic (PAL) and prove that the refinement relation on static models can assure us to safely verify any dynamic properties in terms of PAL-formulas on the abstractions of a KMLTS. Thus the theory can be used to abstract Kripke models, since Kripke models can be regarded as special case of KMLTSs. This theory is in particular applicable for an epistemic setting as the example of the Muddy Children shows.

In the flourishing field of abstraction techniques, to the best of our knowledge, no work on the abstraction of Kripke models exists yet reducing both the number of labels and of basic propositions. The literature related most closely to the current paper is the work on abstraction of LTSs [20] in which the labels could be grouped. Since both temporal and knowledge properties can be expressed using box- and diamond modalities of modal languages, model checkers on LTSs are sometimes employed to verify epistemic properties 111922. However, LTS abstractions were never used in this context. A complementary technique for escaping the epistemic explosion problem is symbolic model checking discussed in [17.

Section 2 introduces Kripke Modal Labelled Transition Systems, together with a 3-valued interpretation of PAL. In Section 3, the notions of refinement and abstraction are introduced and the preservation results are proven. Section 4 contains two examples of applying abstraction to some real epistemic models. We conclude in Section 5 .

\section{Preliminaries}

In this section we introduce the 3 -valued Public Announcement Logic (PAL) interpreted on 3-valued Kripke Modal Labelled Transition Systems.

\subsection{Kripke Modal Labelled Transition System}

A standard Kripke model consists of a set of states $S$, the labelled relations $R$ among them and a 2 -valued valuation $V$ which assigns a truth value to each 
basic proposition in each state1. In order to define abstractions of Kripke models the standard definition is extended in the following sense:

- To incorporate the approximation of propositional information in the abstract model, we use 3 -valued valuations instead of 2 -valued ones. Besides true and false, atomic propositions can now have a third truth value $\perp$ which is intended to mean unknown.

- To incorporate the approximation of relations, two types of relations must and may are introduced as in Modal Transition Systems [16, where must transitions are under-approximations (the relations are necessarily there in the concrete model) and may for over-approximations (there are possibly such relations). Since necessarily existent relations should be at least possible, we require that the must relations are included in the may relations.

Formally, similar to the definition of Kripke Modal Transition Systems in [149], we have:

Definition 1 (Kripke Modal Labelled Transition System). A Kripke Modal Labelled Transition System (KMLTS) is a tuple $\mathcal{M}=\left(I, P ; S, \rightarrow \diamond, \rightarrow_{\square}, V\right)$ where:

- I is a non-empty set of labels;

- $P$ is a set of basic propositions;

- $S$ is a non-empty set of states;

- $\rightarrow \diamond$ is a set of transitions of the form $s \stackrel{i}{\rightarrow} \diamond s^{\prime}$ where $i \in I$;

$-\rightarrow \square$ is a set of transitions of the form $s \stackrel{i}{\rightarrow} s^{\prime}$ where $i \in I$;

$-V$ is a valuation function: $V: S \rightarrow\{\text { true, false, } \perp\}^{P}$.

We require that $\rightarrow \square \subseteq \rightarrow \diamond$. We call $(I, P)$ the signature of $\mathcal{M}$. A pointed KMLTS $(\mathcal{M}, s)$ is a pair of a KMLTS $\mathcal{M}$ and a distinguished state $s$ in it.

We include the signature $(I, P)$ in the specification of the models as, in general, the signatures of a model and its abstraction will be different.

A standard Kripke model can be regarded as a special kind of KMLTS, where must and may coincide and the valuation is essentially 2-valued:

Definition 2 (Concrete model). A KMLTS $\mathcal{M}=\left(I, P ; S, \rightarrow \diamond, \rightarrow_{\square}, V\right)$ is a concrete model if:

$-\rightarrow \diamond=\rightarrow \square$

- for all $s \in S$, all $p \in P: V(s)(p) \neq \perp$.

${ }^{1}$ In an epistemic setting, the states (also called "possible worlds") are interpreted as states of affairs that may be considered possible by agents: an $i$-relation from one state to another means that at the first state agent $i$ considers the second possible. 


\subsection{Public Announcement Logic}

Public Announcement Logic (PAL) initiated in 18 is a convenient language to describe announcements and their informational consequences for (a group of) agents. Based on the standard language of epistemic logic (logic of knowledge), a new modality $[\phi]$ is introduced into the language, with $[\phi] \psi$ intended to express "if $\phi$ is true then after the announcement of $\phi, \psi$ is true.". Various case studies showed this logic to be powerful in helping to understand complicated higher order reasoning about knowledge and announcements such as in the cases of Muddy Children, Sum and Product and the protocol of Dining Cryptographers (we refer interested readers to 21] for detailed explanations).

Formally, given a signature $(I, P)$, the formulas of the Public Announcement Logic $\mathcal{L}_{I, P}$ are defined by

$$
\phi, \psi::=p|\phi \wedge \psi| \neg \phi\left|\square_{i} \phi\right|[\phi] \psi
$$

where $p \in P, i \in I$. As usual, we define $\phi \vee \psi, \phi \rightarrow \psi$ and $\diamond_{i} \phi$ as abbreviations of $\neg(\neg \phi \wedge \neg \psi), \neg \phi \vee \psi$ and $\neg \square_{i} \neg \phi$ respectively.

As we will see in the next section, our overall approach is not constrained to be used only in epistemic settings, as it does not require the model to be $S 52$ Not constrained within $S 5$ models, we will have more freedom to find suitable abstractions, as we will see in the Muddy Children example.

\subsection{Semantics}

The semantics for 2-valued public announcement logic is the extension of standard modal logic with relativization operators $[\phi]: \mathcal{M}, s \vDash[\phi] \psi \Longleftrightarrow[\mathcal{M}, s \vDash \phi$ implies $\left.\left.\mathcal{M}\right|_{\phi}, s \vDash \psi\right]$, where the relativized model $\left.\mathcal{M}\right|_{\phi}$ is the restriction of $\mathcal{M}$ to the states where $\phi$ holds. We extend such relativization, which we call "update" in the context of PAL, to the 3 -valued case and take the usual semantics for $\square$ as in the logics on Modal Transition Systems:

Definition 3 (3-valued Semantics). The truth value of a $\mathcal{L}_{I, P}$ formula $\phi$ in a state $s$ of a KMLTS $\mathcal{M}=(I, P ; S \rightarrow \diamond, \rightarrow \square, V)$, written $\llbracket \phi \rrbracket^{\mathcal{M}, s}$, is defined by:

$$
\begin{aligned}
& \llbracket p \rrbracket^{\mathcal{M}, s}=V(s)(p) \\
& \llbracket \neg \phi \rrbracket^{\mathcal{M}, s}=\neg 3 \llbracket \phi \rrbracket^{\mathcal{M}, s} \\
& \llbracket \phi \wedge \psi \rrbracket^{\mathcal{M}, s}=\llbracket \phi \rrbracket^{\mathcal{M}, s} \wedge_{3} \llbracket \psi \rrbracket^{\mathcal{M}, s} \\
& \llbracket \square_{i} \phi \rrbracket^{\mathcal{M}, s}=\left\{\begin{array}{l}
\text { true if } \forall s^{\prime}: s \stackrel{i}{\rightarrow} s^{\prime} \Longrightarrow \llbracket \phi \rrbracket^{\mathcal{M}, s^{\prime}}=\text { true } \\
\text { false if } \exists s^{\prime}: s \stackrel{i}{\rightarrow} s^{\prime} \text { and } \llbracket \phi \rrbracket^{\mathcal{M}, s^{\prime}}=\text { false } \\
\perp \quad \text { otherwise }
\end{array}\right. \\
& \llbracket[\phi] \psi \rrbracket^{\mathcal{M}, s}=\left\{\begin{array}{l}
\text { true } \text { if } \llbracket \phi \rrbracket^{\mathcal{M}, s}=\text { false or } \llbracket \psi \rrbracket^{\left.\mathcal{M}\right|_{\phi}, s}=\text { true } \\
\text { false } \text { if } \llbracket \phi \rrbracket^{\mathcal{M}, s}=\text { true and } \llbracket \psi \rrbracket^{\left.\mathcal{M}\right|_{\phi}, s}=\text { false } \\
\perp \quad \text { otherwise }
\end{array}\right.
\end{aligned}
$$

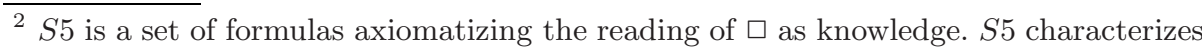
models in which the relations are equivalence relations. 
where:

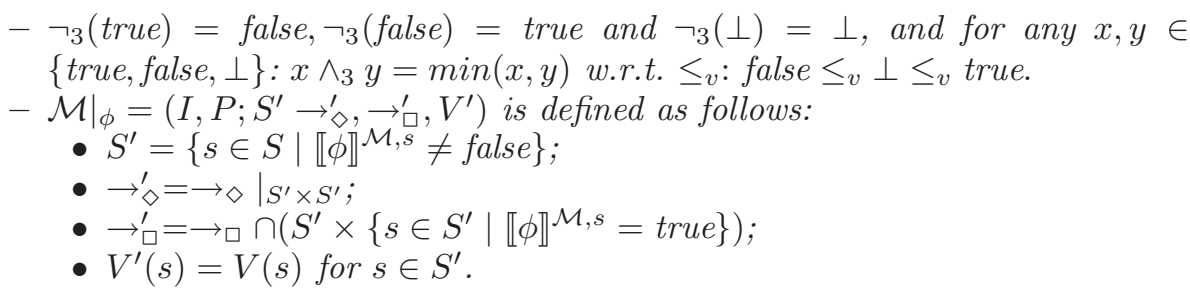

The intuitive idea behind the semantics of $\square$ is that $\square \phi$ is true if all the possible ( may) relations lead to $\phi$-true states, and is false if there exists a necessary ( $m u s t$ ) relation leading to a $\phi$-false state.

The updated model $\left.\mathcal{M}\right|_{\phi}$ keeps all $\phi$-not-false states and all the relations among them, except for the must relations directed at a $\phi$-unknown state 3 Note that $\left.\mathcal{M}\right|_{\phi}$ is still a KMLTS since $\rightarrow_{\square}^{\prime} \subseteq \rightarrow_{\diamond}^{\prime}$ by definition. It is not hard to check that this three valued semantics "coincides" with the standard 2-valued semantics on concrete models. Formally, for any $\mathcal{L}_{I, P}$ formula $\phi$, any concrete model $\mathcal{M}$ :

$$
\llbracket \phi \rrbracket^{\mathcal{M}, s}=\text { true } \Longleftrightarrow \mathcal{M}^{\prime}, s \vDash \phi \quad \llbracket \phi \rrbracket^{\mathcal{M}, s}=\text { false } \Longleftrightarrow \mathcal{M}^{\prime}, s \not \models \phi
$$

where $\mathcal{M}^{\prime}$ is the standard Kripke model converted from $\mathcal{M}$ by lumping may and must relations together. For 2-valued Public Announcement Logic the following reduction axioms hold:

$$
\begin{array}{rlll}
\text { (At) } & {[\phi] p} & \leftrightarrow & \leftrightarrow \\
\text { (PF) } & {[\phi] \neg \psi} & \leftrightarrow & \leftrightarrow \rightarrow \neg[\phi] \psi \\
\text { (Dist) }[\phi]\left(\psi_{1} \wedge \psi_{2}\right) & \leftrightarrow & \leftrightarrow \phi] \psi_{1} \wedge[\phi] \psi_{2} \\
\text { (Seq) }[\phi][\psi] \chi & \leftrightarrow & \leftrightarrow \phi \wedge[\phi] \psi] \chi \\
\text { (KA) } \quad[\phi] \square_{i} \psi & \leftrightarrow \phi \rightarrow \square_{i}[\phi] \psi
\end{array}
$$

In the 3 -valued case, there are a few cases where the left hand side of $\leftrightarrow$ gives false while the right hand side gives $\perp$, all involving the valuation of $\phi$ to be $\perp$. So if we only consider concrete models then the evaluation of $\phi$ is either true or false and the above equivalences hold.

Although our concern in this paper is primarily to develop the theory of epistemic abstrcations, the ultimate goal is to enable automatic verification of large epistemic models. Designing efficient algorithms for checking the satisfaction of 3-valued PAL formulae on KLMTSs, based on the definition above, is an interesting topic in itself and we leave it as further work. We now only note that, looking at similar results in the literature [3], it is to expect that such a model checking algorithm will not be more complex than the ones for checking (2-valued) PAL on KMs or LTSs.

\footnotetext{
3 The must-relations signify necessary relations. However, a $\phi$-unknown state $s$ is not necessarily there in the updated model, as unknown leaves the possibility open that $\phi$ could 'actually' be false, in which case $s$ would not be in the updated model. A relation directed at a possibly but not necessarily existent state, cannot be a necessary relation, so must-relations to $\phi$-unknown states are removed.
} 


\section{Refinement and Logical Characterization}

In this section we extend the classic definition of refinement with label and proposition mapping in order to reduce the number of labels and possibly achieve smaller abstraction models. We show that we can reason about properties of the more refined model by model checking the more abstract model.

\subsection{Refinement and Abstraction}

As observed in 20], to do model checking on infinitely-labelled systems, one needs abstraction to obtain a model with a reduced number of labels. We aim for an abstraction method to reduce the labels also in the finite case, by lumping similar transitions with different labels together into a unified one. This is often applicable in the epistemic case, as several agents may play a similar role and therefore have similar uncertainties. On the other hand, different propositions may also have a similar role on different states, in which case abstractions may combine propositions together as well. In the following, we use two mappings from one signature to the other to capture the above intuitions of lumping labels and propositions. It is important to note that these abstractions produce models with a different signature.

Notation For a function $h$ and $x$ in its range, we use $h^{-1}[x]$ to denote the preimage of $x$.

Definition 4 (Refinement and Abstraction). Given two KMLTSs $\mathcal{M}=$ $\left(I, P ; S, \rightarrow_{\diamond, \rightarrow \square}, V\right)$ and $\mathcal{N}=\left(I^{\prime}, P^{\prime} ; T, \rightarrow_{\diamond}^{\prime}, \rightarrow_{\square}^{\prime}, V^{\prime}\right)$ and two surjective functions $f: I^{\prime} \rightarrow I$ and $g: P^{\prime} \rightarrow P$, a binary relation $R \subseteq T \times S$ is called an $f, g$-refinement relation between $\mathcal{N}$ and $\mathcal{M}$, if for all $t \in T, s \in S$ with $(t, s) \in R$ the following hold:

- for any $p \in P: V(s)(p) \neq \perp$ implies for all $p^{\prime} \in g^{-1}[p]: V^{\prime}(t)\left(p^{\prime}\right)=V(s)(p)$;

$-t \stackrel{i^{\prime}}{\rightarrow} t^{\prime}$ implies $\exists s^{\prime} \in S: s \stackrel{f\left(i^{\prime}\right)}{\rightarrow} s^{\prime}$ and $R\left(t^{\prime}, s^{\prime}\right)$

$-s \stackrel{i}{\rightarrow} s^{\prime}$ implies $\forall i^{\prime} \in f^{-1}[i]: \exists t^{\prime} \in T$ such that $t \stackrel{i^{\prime}}{\rightarrow} t^{\prime}$ and $R\left(t^{\prime}, s^{\prime}\right)$.

$W e$ say $\mathcal{N}$ is a $f, g$-refinement of $\mathcal{M}$ (notation: $\mathcal{N} \Subset_{f, g} \mathcal{M}$ ) if there exists an $f, g$ refinement relation $R$ between $\mathcal{N}$ and $\mathcal{M}$. We say $(\mathcal{N}, t)$ is an $f, g$-refinement of $(\mathcal{M}, s)$ (notation: $\left.(\mathcal{N}, t) \Subset_{f, g}(\mathcal{M}, s)\right)$ if there exists an $f, g$-refinement relation $R$ between $\mathcal{N}$ and $\mathcal{M}$ such that $(t, s) \in R$.

Correspondingly, $(\mathcal{M}, s)$ is called an $f, g$-abstraction of $(\mathcal{N}, t)$ iff $(\mathcal{N}, t)$ is an $f, g$-refinement of $(\mathcal{M}, s)$.

The first condition says that the valuation in the more abstract model can be less informative by making some propositions unknown $(\perp)$, but never unfaithful. The intuition behind the requirement of must is that an $i$-must relation in the more abstract model is like an intersection of corresponding $i^{\prime}$-must for $i^{\prime} \in$ $f^{-1}[i]$. For may, an $f\left(i^{\prime}\right)$-may relation in the more abstract model is like a union of those $i^{\prime \prime}$-may relations in the more refined model for which $f\left(i^{\prime \prime}\right)=f\left(i^{\prime}\right)$. 


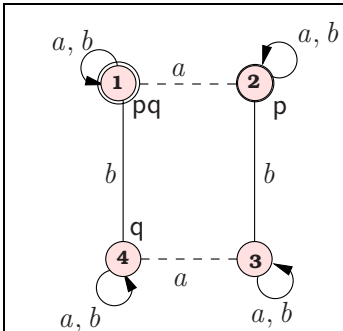

(1) KMLTS $\mathcal{M}$

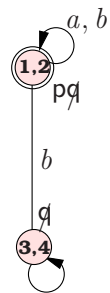

$a, b$

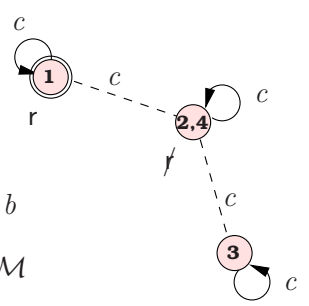

(4) $(\{a, b\} \mapsto c,\{\mathrm{p}, \mathrm{q}\} \mapsto \mathrm{r})$

(2) Id, Id-abstraction of $\mathcal{M}$

Fig. 1. A pointed KMLTS and three possible abstractions of it. Dot lines are for may relations and solid lines for must. May relations that coincide with corresponding must ones are omitted. If there is no arrow on a relation then it is bidirectional. $\not p$ is to mean the value of $p$ is unknown $(\perp)$ at the current state. For clarity, the states of $\mathcal{M}$ are numbered and the numbers on the states of the abstracted models indicate which original states they represent. In (2), the mappings are the identity functions, and the valuation of proposition $\mathrm{q}$ is mapped to $\perp$ for all worlds. In (3), the abstraction is given by the identity functions as well, but collapsing different worlds. In (4), there's an abstraction obtained by lumping both agents and both propositions.

Note that for two 2-valued Kripke models with the same signature $(I, P), \mathcal{N}$ is a refinement of $\mathcal{M}$ in the classical sense of [15] iff $\mathcal{N}$ is an $\left(I d_{I}, I d_{P}\right)$-refinement of $\mathcal{M}$ where $I d_{X}$ is identity function on the domain $X$.

Fig. 1] shows an example of a KMLTS $\mathcal{M}$ and some abstractions of it.

Since $\rightarrow \square \subseteq \rightarrow \diamond$, we can make a concrete refinement of any KMLTS by dropping may relations that do not have a must counterpart (i.e. $\rightarrow_{\diamond}^{\prime}, \rightarrow_{\square}^{\prime}:=\rightarrow \square$ ) and by adapting the valuation to become two-valued (e.g. by defining $V^{\prime}(s)(p)=$ false whenever $V(s)(p)=\perp$ and $V^{\prime}(s)(p)=V(s)(p)$ otherwise). Therefore:

Proposition 1. A KMLTS $\mathcal{M}$ always has a concrete refinement.

\subsection{Logical Characterization}

We will prove a preservation result of satisfaction of formulas between a pointed model $(\mathcal{N}, t)$ and its abstraction $(\mathcal{M}, s)$. Intuitively we want a formula to be true/false at $\mathcal{N}$ if it is true/false at $\mathcal{M}$ respectively, such that we can safely model check the more abstract model to get the information of the more refined one. However, as these models may have different signatures due to the $f, g$ mappings attached to the refinement relation, we need to check different formulas on these two models. Given two pointed models $(\mathcal{M}, s),(\mathcal{N}, t)$, and two formulas $\phi, \psi$, we say $\llbracket \psi \rrbracket^{\mathcal{M}, s} \leq \llbracket \phi \rrbracket^{\mathcal{N}, t}$ if the following hold:

1. $\llbracket \psi \rrbracket^{\mathcal{M}, s}=$ true $\Longrightarrow \llbracket \phi \rrbracket^{\mathcal{N}, t}=$ true;

2. $\llbracket \psi \rrbracket^{\mathcal{M}, s}=$ false $\Longrightarrow \llbracket \phi \rrbracket^{\mathcal{N}, t}=$ false. 
Then our goal is to check whether $(\mathcal{N}, t) \Subset_{f, g}(\mathcal{M}, s)$ implies for all $\phi$ : $\llbracket\ulcorner\phi\urcorner \rrbracket^{\mathcal{M}, s} \leq \llbracket \phi \rrbracket^{\mathcal{N}, t}$ where $\ulcorner\phi\urcorner$ is a formula in the signature of $\mathcal{M}$ corresponding to $\phi$. To pinpoint the right formulas to check, we introduce the following translation:

Definition 5 (Translation of formulas). Given signatures $\left(I^{\prime}, P^{\prime}\right),(I, P)$, and surjective functions $f: I^{\prime} \rightarrow I, g: P^{\prime} \rightarrow P$, we define the translation of an $\mathcal{L}_{I^{\prime}, P^{\prime}}$-formula $\phi$ into an $\mathcal{L}_{I, P}$-formula $\ulcorner\phi\urcorner_{f, g}$ inductively as follows:

$$
\begin{array}{ll}
\left\ulcorner p^{\prime}\right\urcorner_{f, g} & =g\left(p^{\prime}\right) \\
\ulcorner\neg \psi\urcorner_{f, g} & =\neg\ulcorner\psi\urcorner_{f, g} \\
\left\ulcorner\psi_{1} \wedge \psi_{2}\right\urcorner_{f, g} & =\left\ulcorner\psi_{1}\right\urcorner f, g \wedge\left\ulcorner\psi_{2}\right\urcorner_{f, g} \\
\left\ulcorner\square_{i^{\prime}}\right\urcorner_{f, g} & =\square_{f\left(i^{\prime}\right)}\ulcorner\psi\urcorner_{f, g} \\
\ulcorner[\chi] \psi\urcorner_{f, g} & =\left[\ulcorner\chi\urcorner_{f, g}\right]\ulcorner\psi\urcorner_{f, g}
\end{array}
$$

Before proving the main result of this paper, we first prove a result establishing the refinement relation between the updated models $\left(\left.\mathcal{N}\right|_{\chi}, t\right)$ and $\left(\left.\mathcal{M}\right|_{\ulcorner\chi\urcorner_{f, g}}, s\right)$ for some $\mathcal{L}_{I, P}$-formula $\chi$, given that $(\mathcal{N}, t) \Subset_{f, g}(\mathcal{M}, s)$

Lemma 1. Suppose $(\mathcal{N}, t),(\mathcal{M}, s)$ are pointed $K M L T S s$ with signatures $\left(I^{\prime}, P^{\prime}\right)$ and $(I, P)$ and set of states $T$ and $S$ respectively, such that $(\mathcal{N}, t) \Subset_{f, g}(\mathcal{M}, s)$. Then for any $\mathcal{L}_{I^{\prime}, P^{\prime}}$ formula $\chi$ such that $t \in \mathcal{N} \mid \chi$ and $s \in \mathcal{M} \mid\ulcorner\chi\urcorner_{f, g}$, we have $\left(\left.\mathcal{N}\right|_{\chi}, t\right) \Subset_{f, g}\left(\left.\mathcal{M}\right|_{\ulcorner\chi\urcorner_{f, g}}, s\right)$ if for each $t^{\prime} \in T, s^{\prime} \in S$ the following condition holds:

$$
\left(\mathcal{N}, t^{\prime}\right) \Subset_{f, g}\left(\mathcal{M}, s^{\prime}\right) \Longrightarrow \llbracket\ulcorner\chi\urcorner f, g \rrbracket^{\mathcal{M}, s^{\prime}} \leq \llbracket \chi \rrbracket^{\mathcal{N}, t^{\prime}}
$$

Proof. Suppose $(\mathcal{N}, t) \Subset_{f, g}(\mathcal{M}, s)$ then there is a relation $R$ which constitutes an $f, g$-refinement between $\mathcal{N}$ and $\mathcal{M}$ with $(t, s) \in R$. We claim that $R^{\prime}=$ $R \cap\left(\left.\mathcal{N}\right|_{\chi} \times\left.\mathcal{M}\right|_{\ulcorner\chi\urcorner_{f, g}}\right)$ is an $f, g$-refinement relation between $\left.\mathcal{N}\right|_{\chi}$ and $\left.\mathcal{M}\right|_{\ulcorner\chi\urcorner_{f, g}}$. Note that $(t, s) \in R^{\prime}$ since $\left.t \in \mathcal{N}\right|_{\chi}$ and $\left.\left.s \in \mathcal{M}\right|_{\ulcorner\chi\urcorner}\right\urcorner_{f, g}$. Now we check the three conditions of the refinement relation:

- for the condition on $p$ : follows from this property of $R$ and the fact that the valuation of an updated model is just the restriction of the original valuation to the remaining states.

- Suppose $t \stackrel{i^{\prime}}{\rightarrow} \diamond t^{\prime}$ in $\left.\mathcal{N}\right|_{\chi}$, then $t \stackrel{i^{\prime}}{\rightarrow} \diamond t^{\prime}$ in $\mathcal{N}$ according to the definition of the update. Since $(\mathcal{N}, t) \Subset_{f, g}(\mathcal{M}, s)$, there exists $s^{\prime} \in \mathcal{M}: s \stackrel{f\left(i^{\prime}\right)}{\rightarrow} s^{\prime}$ and $\left(t^{\prime}, s^{\prime}\right) \in R$. Remains to show that $\left.s^{\prime} \in \mathcal{M}\right|_{\ulcorner\chi\urcorner_{f, g}}$. Suppose not, then $\llbracket\ulcorner\chi\urcorner_{f, g} \rrbracket^{\mathcal{M}, s^{\prime}}=$ false. Because $\left(t^{\prime}, s^{\prime}\right) \in R$ ensures $\left(\mathcal{N}, t^{\prime}\right) \Subset_{f, g}\left(\mathcal{M}, s^{\prime}\right)$, it then follows from condition $(\star)$ that $\llbracket \chi \rrbracket^{\mathcal{N}, t^{\prime}}=$ false. But then $\left.t^{\prime} \notin \mathcal{N}\right|_{\chi}$, contradiction.

- Suppose $s \stackrel{i}{\rightarrow} \square s^{\prime}$ in $\left.\mathcal{M}\right|_{\ulcorner\chi\urcorner_{f, g}}$, then $\llbracket\ulcorner\chi\urcorner_{f, g} \rrbracket^{\mathcal{M}, s^{\prime}}=$ true and $s \stackrel{i^{\prime}}{\rightarrow} s^{\prime}$ in $\mathcal{M}$. Because $R$ is an $f, g$-refinement between $(\mathcal{N}, t)$ and $(\mathcal{M}, s)$, for any $i^{\prime} \in f^{-1}[i]$ there exists $t^{\prime} \in \mathcal{N}$ such that $t \stackrel{i^{\prime}}{\rightarrow} \square t^{\prime}$ and $\left(t^{\prime}, s^{\prime}\right) \in R$. To show that $\left(t^{\prime}, s^{\prime}\right) \in$ $R^{\prime}$ for such $t^{\prime}$, it remains to show that $\left.t^{\prime} \in \mathcal{N}\right|_{\chi}$. Since $\llbracket\ulcorner\chi\urcorner f, g \rrbracket^{\mathcal{M}, s^{\prime}}=$ true and $\left(t^{\prime}, s^{\prime}\right) \in R$, it then follows from condition $(\star)$ that $\llbracket \chi \rrbracket^{\mathcal{N}, t^{\prime}}=$ true. Hence, $\left.t^{\prime} \in \mathcal{N}\right|_{\chi}$. 
Theorem 1. Suppose $\mathcal{N}, \mathcal{M}$ are KMLTSs w.r.t. $I^{\prime}, P^{\prime}$ and $I, P$ respectively. $s$ and $t$ are two worlds in $\mathcal{M}$ and $\mathcal{N}$ respectively. Then $(\mathcal{N}, t) \Subset_{f, g}(\mathcal{M}, s)$ implies for all $\phi \in \mathcal{L}_{I^{\prime}, P^{\prime}}: \llbracket\ulcorner\phi\urcorner f, g \rrbracket^{\mathcal{M}, s} \leq \llbracket \phi \rrbracket^{\mathcal{N}, t}$.

Proof. We prove the theorem by induction on the structure of $\phi$ :

$-\phi=p^{\prime}$ : trivial, follows from the first condition of the definition of refinement.

$-\phi=\neg \psi$ : suppose $\llbracket\ulcorner\phi\urcorner_{f, g} \rrbracket^{\mathcal{M}, s}=$ true then according to the semantics

$\llbracket\ulcorner\psi\urcorner f, g \rrbracket^{\mathcal{M}, s}=$ false. Thus by induction hypothesis $\llbracket \psi \rrbracket^{\mathcal{N}, t}=$ false. Therefore $\llbracket \phi \rrbracket^{\mathcal{N}, t}=$ true. For the case $\llbracket\ulcorner\phi\urcorner f, g \rrbracket^{\mathcal{M}, s}=$ false, similar.

$-\phi=\psi_{1} \wedge \psi_{2}$ :

- suppose $\llbracket\ulcorner\phi\urcorner_{f, g} \rrbracket^{\mathcal{M}, s}=$ true then by the semantics: $\llbracket\left\ulcorner\psi_{1}\right\urcorner_{f, g} \rrbracket^{\mathcal{M}, s}=$ true and $\llbracket\left\ulcorner\psi_{2}\right\urcorner f, g \rrbracket^{\mathcal{M}, s}=$ true. Thus by induction hypothesis $\llbracket \psi_{1} \rrbracket^{\mathcal{N}, t}=$ true and $\llbracket \psi_{2} \rrbracket^{\mathcal{N}, t}=$ true. Therefore $\llbracket \phi \rrbracket^{\mathcal{N}, t}=$ true.

- suppose $\llbracket\ulcorner\phi\urcorner f, g \rrbracket^{\mathcal{M}, s}=$ false then by the semantics either $\llbracket\left\ulcorner\psi_{1}\right\urcorner f, g \rrbracket^{\mathcal{M}, s}=$ false or $\llbracket\left\ulcorner\psi_{2}\right\urcorner_{f, g} \rrbracket^{\mathcal{M}, s}=$ false. Without loss of generality, suppose the latter. Thus by induction hypothesis $\llbracket \psi_{2} \rrbracket^{\mathcal{N}, t}=$ false. Therefore $\llbracket \phi \rrbracket^{\mathcal{N}, t}=$ false.

- $\phi=\square_{i^{\prime}} \psi$ : then $\ulcorner\phi\urcorner_{f, g}=\square_{f\left(i^{\prime}\right)}\ulcorner\psi\urcorner_{f, g}$.

- suppose $\llbracket\ulcorner\phi\urcorner f, g \rrbracket^{\mathcal{M}, s}=$ true then according to the semantics for all $s^{\prime}$ with $s \stackrel{f\left(i^{\prime}\right)}{\rightarrow \diamond} s^{\prime}$ we have $\llbracket\ulcorner\psi\urcorner f, g \rrbracket^{\mathcal{M}, s^{\prime}}=$ true. Suppose in $\mathcal{N}$ there is a world $t^{\prime}$ such that $t \stackrel{i^{\prime}}{\rightarrow} \diamond t^{\prime}$ then according to the definition of refinement, there is a $s^{\prime \prime} \in \mathcal{M}$ such that $s \stackrel{f\left(i^{\prime}\right)}{\rightarrow} s^{\prime \prime}$ and $\left(\mathcal{N}, t^{\prime}\right) \Subset_{f, g}\left(\mathcal{M}, s^{\prime \prime}\right)$. Thus $\llbracket\ulcorner\psi\urcorner_{f, g} \rrbracket^{\mathcal{M}, s^{\prime \prime}}=$ true. By induction hypothesis, $\llbracket \psi \rrbracket^{\mathcal{N}, t^{\prime}}=$ true. Therefore $\llbracket \square_{i^{\prime}} \psi \rrbracket^{\mathcal{N}, t}=$ true.

- suppose $\llbracket\ulcorner\phi\urcorner_{f, g} \rrbracket^{\mathcal{M}, s}=$ false then according to the semantics, there is $s^{\prime}$ with $s \stackrel{f\left(i^{\prime}\right)}{\rightarrow} s^{\prime}$ such that $\llbracket\ulcorner\psi\urcorner f, g \rrbracket^{\mathcal{M}, s}=$ false. By definition of refinement, for any $i^{\prime \prime} \in f^{-1}\left[f\left(i^{\prime}\right)\right]$ there is a $t^{\prime} \in \mathcal{N}$ such that $t \stackrel{i^{\prime \prime}}{\rightarrow} t^{\prime}$ and $\left(\mathcal{N}, t^{\prime}\right) \Subset_{f, g}$ $\left(\mathcal{M}, s^{\prime}\right)$. By induction hypothesis, for all such $t^{\prime}: \llbracket \psi \rrbracket^{\mathcal{N}, t^{\prime}}=$ false. Thus for all $i^{\prime \prime} \in f^{-1}\left[f\left(i^{\prime}\right)\right]: \llbracket \square_{i^{\prime \prime}} \psi \rrbracket^{\mathcal{N}, t}=$ false. In particular: $\llbracket \square_{i^{\prime}} \psi \rrbracket^{\mathcal{N}, t}=$ false.

$-\phi=[\chi] \psi$

- if $\llbracket\ulcorner\phi\urcorner f, g \rrbracket^{\mathcal{M}, s}=$ true then $\llbracket\ulcorner\chi\urcorner f, g \rrbracket^{\mathcal{M}, s}=$ false or $\llbracket\ulcorner\psi\urcorner_{f, g} \rrbracket^{\mathcal{M} \mid\left\ulcorner_{\chi}\right\urcorner_{f, g}, s}=$ true. If $\llbracket\ulcorner\chi\urcorner f, g \rrbracket^{\mathcal{M}, s}=$ false then $\llbracket \chi \rrbracket^{\mathcal{N}, t}=$ false by induction hypothesis, hence $\llbracket \phi \rrbracket^{\mathcal{N}, t}=$ true. Otherwise, $\llbracket\ulcorner\psi\urcorner_{f, g} \rrbracket^{\left.\left.\mathcal{M}\right|_{\ulcorner\chi\urcorner}\right\urcorner_{f, g}, s}=$ true and $\llbracket\ulcorner\chi\urcorner_{f, g} \rrbracket^{\mathcal{M}, s} \neq$ false, so $\left.s \in \mathcal{M}\right|_{\ulcorner\chi\urcorner_{f, g}}$. Now suppose $\llbracket \chi \rrbracket^{\mathcal{N}, t} \neq$ false, so: $\left.t \in \mathcal{N}\right|_{\chi}$. We need to show that $\left.\llbracket \psi \rrbracket^{\mathcal{N}}\right|_{\chi}, t=t r u e$. By induction hypothesis $\left(\mathcal{N}, t^{\prime}\right) \Subset_{f, g}\left(\mathcal{M}, s^{\prime}\right) \Longrightarrow \llbracket\ulcorner\chi\urcorner_{f, g} \rrbracket^{\mathcal{M}, s^{\prime}} \leq \llbracket \chi \rrbracket^{\mathcal{N}, t^{\prime}}$ for each $s^{\prime} \in S, t^{\prime} \in T$. Therefore from Lemma 1 we have $\left(\left.\mathcal{N}\right|_{\chi}, t\right) \Subset_{f, g}\left(\left.\mathcal{M}\right|_{\ulcorner\chi\urcorner_{f, g}}, s\right)$. By induction hypothesis, $\left.\llbracket \psi \rrbracket^{\mathcal{N}}\right|_{\chi}, t=$ true. Thus $\llbracket \phi \rrbracket^{\mathcal{N}, t}=$ true.

- if $\llbracket\ulcorner\phi\urcorner_{f, g} \rrbracket^{\mathcal{M}, s}=$ false then $\llbracket\ulcorner\chi\urcorner_{f, g} \rrbracket^{\mathcal{M}, s}=$ true and $\llbracket\ulcorner\psi\urcorner_{f, g} \rrbracket^{\left.\left.\mathcal{M}\right|_{\ulcorner\chi\urcorner}\right\urcorner_{f, g}, s}=$ false. Since $\llbracket\ulcorner\chi\urcorner_{f, g} \rrbracket^{\mathcal{M}, s}=$ true then $\llbracket \chi \rrbracket^{\mathcal{N}, t}=$ true by induction hypothesis. We only need to show $\left.\llbracket \psi \rrbracket^{\mathcal{N}}\right|_{\chi}, s=$ false. It is clear that $\left.t \in \mathcal{N}\right|_{\chi}$ and $\left.s \in \mathcal{M}\right|_{\ulcorner\chi\urcorner_{f, g}}$, then by the induction hypothesis the condition of Lemma1 holds, and it follows that $\left(\left.\mathcal{N}\right|_{\chi}, t\right) \Subset_{f, g}\left(\left.\mathcal{M}\right|_{\ulcorner\chi\urcorner_{f, g}}, s\right)$. Thus by the induction hypothesis we have $\llbracket \psi \rrbracket^{\left.N\right|_{\chi}, t}=$ false. Therefore: $\llbracket \phi \rrbracket^{\mathcal{N}, t}=$ false. 
Corollary 1. Suppose $(\mathcal{N}, t),(\mathcal{M}, s)$ are two pointed KMLTSs w.r.t. $\left(I^{\prime}, P^{\prime}\right)$ and $(I, P)$ respectively. If $(\mathcal{N}, t) \Subset_{f, g}(\mathcal{M}, s)$ and $\mathcal{N}$ is a Kripke model converted from a concrete KMLTS then for any formula $\phi \in \mathcal{L}_{I^{\prime}, P^{\prime}}$ :

$-\llbracket\ulcorner\phi\urcorner_{f, g} \rrbracket^{\mathcal{M}, s}=$ true $\Longrightarrow \mathcal{N}, t \vDash \phi$

$-\llbracket\ulcorner\phi\urcorner_{f, g} \rrbracket^{\mathcal{M}, s}=$ false $\Longrightarrow \mathcal{N}, t \vDash \neg \phi$

By the above corollary, to know whether $\phi$ is satisified at a pointed Kripke model, we can instead model check $\ulcorner\phi\urcorner_{f, g}$ on its $f, g$-abstraction.

To justify the logical characterization, we prove the converse of Theorem 1 .

Theorem 2. Suppose $(\mathcal{N}, t)$ and $(\mathcal{M}, s)$ are pointed KMLTS models with signatures $\left(I^{\prime}, P^{\prime}\right)$ and $(I, P)$, and suppose they enjoy image finiteness (i.e. every transition relation has most finitely many successors at any state). If for every formula $\phi \in \mathcal{L}_{I^{\prime}, P^{\prime}}: \llbracket\ulcorner\phi\urcorner_{f, g} \rrbracket^{\mathcal{M}, s} \leq \llbracket \phi \rrbracket^{\mathcal{N}, t}$ then $(\mathcal{N}, t) \Subset_{f, g}(\mathcal{M}, s)$.

Proof. Assume: for every formula $\phi \in \mathcal{L}_{I^{\prime}, P^{\prime}}: \llbracket\ulcorner\phi\urcorner_{f, g} \rrbracket^{\mathcal{M}, s} \leq \llbracket \phi \rrbracket^{\mathcal{N}, t}$, and let $R=\left\{\left(t^{\prime}, s^{\prime}\right) \mid\right.$ for every $\left.\phi: \llbracket\ulcorner\phi\urcorner f, g \rrbracket^{\mathcal{M}, s^{\prime}} \leq \llbracket \phi \rrbracket^{\mathcal{N}, t^{\prime}}\right\}$. Then $(t, s) \in R$, and we check the three conditions of definition 4 for $R$. Suppose $\left(t^{\prime}, s^{\prime}\right) \in R$, then:

- The first condition follows from $\llbracket\left\ulcorner p^{\prime}\right\urcorner_{f, g} \rrbracket^{\mathcal{M}, s^{\prime}} \leq \llbracket p^{\prime} \rrbracket^{\mathcal{N}, t^{\prime}}$ for $p^{\prime} \in P^{\prime}$.

- Suppose towards contradiction that $\exists t^{\prime \prime}: t^{\prime} \stackrel{i^{\prime}}{\rightarrow} t^{\prime \prime}$ in $\mathcal{N}$ but for any $s^{\prime \prime} \in S$ : $s^{\prime} \stackrel{f\left(i^{\prime}\right)}{\rightarrow} s^{\prime \prime}$ implies $\left(t^{\prime \prime}, s^{\prime \prime}\right) \notin R$. According to image finiteness, we have only finitely many such $s^{\prime \prime}$; call them $s_{0}^{\prime \prime} \ldots s_{n}^{\prime \prime}$. For each $s_{k}^{\prime \prime}$, since $\left(t^{\prime \prime}, s_{k}^{\prime \prime}\right) \notin R$, there must be a formula $\psi_{s_{k}^{\prime \prime}}$ such that $\llbracket\left\ulcorner\psi_{s_{k}^{\prime \prime}}\right\urcorner f, g \rrbracket^{\mathcal{M}, s_{k}^{\prime \prime}}=$ true but $\llbracket \psi_{s_{k}^{\prime \prime}} \rrbracket^{\mathcal{N}, t^{\prime}} \neq$ true 4 Now $\square_{f\left(i^{\prime}\right)}\left(\bigvee_{k=0}^{n}\left\ulcorner\psi_{s_{k}^{\prime \prime}}\right\urcorner f, g\right)$ is true at $s^{\prime}$ but $\square_{i^{\prime}}\left(\bigvee_{k=0}^{n} \psi_{s_{k}^{\prime \prime}}\right)$ is not true at $t^{\prime}$, contradicting the assumption that $\left(t^{\prime}, s^{\prime}\right) \in R$.

- Suppose towards contradiction that $s^{\prime} \stackrel{f\left(i^{\prime}\right)}{\rightarrow} s^{\prime \prime}$ in $\mathcal{M}$, but there exists $i^{\prime \prime} \in f^{-1}\left[f\left(i^{\prime}\right)\right]$ such that $\forall t^{\prime \prime} \in T: t^{\prime} \stackrel{i^{\prime \prime}}{\rightarrow} \square t^{\prime \prime}$ implies $\left(t^{\prime \prime}, s^{\prime \prime}\right) \notin R$. According to image finiteness, there are only finitely many such $t^{\prime \prime}$; call them $t_{0}^{\prime \prime} \ldots t_{n}^{\prime \prime}$. For each $t_{k}^{\prime \prime}$, since $\left(t_{k}^{\prime \prime}, s^{\prime \prime}\right) \notin R$, there must be a formula $\psi_{t_{k}^{\prime \prime}}$ such that $\llbracket\left\ulcorner\psi_{t_{i}^{\prime \prime}}\right\urcorner_{f, g} \rrbracket^{\mathcal{M}, s^{\prime \prime}}=$ false but $\llbracket \psi_{t_{i}^{\prime \prime}} \rrbracket^{\mathcal{N}, t_{i}^{\prime \prime}} \neq$ false. Note that $\square_{f\left(i^{\prime}\right)}\left(\bigvee_{k=0}^{n}\left\ulcorner\psi_{t_{i}^{\prime \prime}}\right\urcorner_{f, g}\right)$ is false at $s^{\prime}$ but $\square_{i^{\prime \prime}}\left(\bigvee_{k=0}^{n} \psi_{t_{i}^{\prime}}\right)$ is not false at $t^{\prime}$, contradicting the assumption that $\left(t^{\prime}, s^{\prime}\right) \in R$.

\section{Examples}

\subsection{The Muddy Children}

A standard example demonstrating the effect of updates on the knowledge within a group of agents, is the epistemic modelling of the Muddy Children Puzzle (cf. the seminal work on reasoning about knowledge [8]). The setting is as follows: out of $n$ children, $k>1$ got mud on their foreheads while playing. They can see

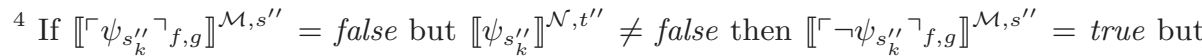
$\llbracket \neg \psi_{s_{k}^{\prime \prime}} \rrbracket^{\mathcal{N}, t^{\prime \prime}} \neq$ true.
} 


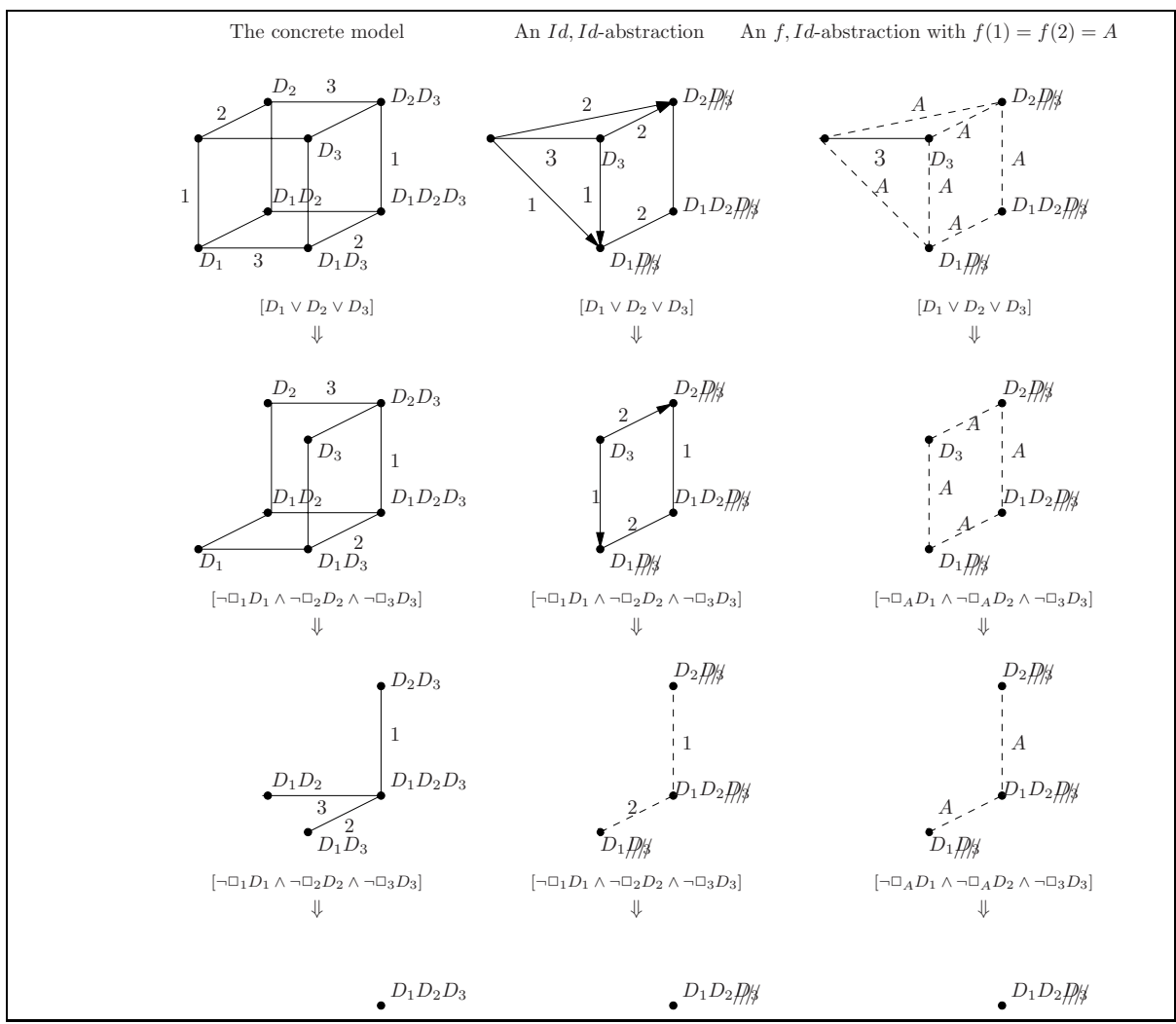

Fig. 2. Abstractions of the Muddy Children for $n=3$ children. Each world has reflexive may-relations for each $i \in I$, some have reflexive must-relations, but for simplicity of presentation, all reflexive relations are omitted; $\not / p / \phi$ means proposition $D_{3}$ has valuation $\perp$ in the current state.

whether other kids are dirty, but there is no mirror for them to discover whether they are dirty themselves. Then father walks in and states: "At least one of you is dirty!" Then he requests "If you know you are dirty, step forward now." If nobody steps forward, he repeats his request: "If you now know you are dirty, step forward now." After exactly $k$ requests to step forward, the $k$ dirty children suddenly do so (assuming they are honest and perfect reasoners).

The left column of Fig. 2 shows the standard epistemic model for this setting with three children. Proposition $D_{i}$ signifies "child $i$ is dirty". After the first update formula ("At least one of you is dirty"), all updates are of the form "nobody knows (yet) he is dirty" (by showing no move). One can check that if only one child is dirty, it will know after the first update. In that case a world satisfying only one $D_{i}$ is the actual world; from this world in the updated model, child $i$ considers no other worlds possible anymore. If nobody steps forward after the first request (implying nobody knows yet whether he is dirty), and a child 
sees only one other muddy child, it will know that he himself must be dirty as well (otherwise this other child would have known previously). This is modelled by the fact that after the second update the worlds with only one dirty child disappear in the updated model (they are no longer considered possible by anybody). If then nothing happens (third update), it must be the case that all three are dirty (and everybody knows this).

The middle and right columns of Fig. 2 show abstracted versions of the concrete model on the left. The refinement relation underlying both abstractions relates three pairs of worlds in the concrete model to three single worlds in the abstraction, while the world with all propositions false and the world with only $D_{3}$ true are kept (for example, the world with $D_{2}$ true and the world with $D_{2}, D_{3}$ true in the concrete model are related to the one world in the abstracted model where $D_{2}$ is true and $D_{3}$ unknown). In the middle column, the parameters $f, g$ for the refinement are identities, in the right column $f$ maps both 1 and 2 to abstract label $A$. Let $D$ be the abbreviation of the first update $\left(D_{1} \vee D_{2} \vee D_{3}\right)$ and $K$ be the abbreviation of the next ones $\left(\neg \square_{1} D_{1} \wedge \neg \square_{2} D_{2} \wedge \neg \square_{3} D_{3}\right)$. Notice the following significant properties can be verified to be true in the two abstractions: (1) In both abstractions, $\left\ulcorner[D][K][K]\left(\square_{1} D_{1} \wedge \square_{2} D_{2}\right)\right\urcorner_{f, g}$ is true at the worlds that correspond to the world which makes $D_{1}, D_{2}$ and $D_{3}$ true in the original model. Thus $[D][K][K]\left(\square_{1} D_{1} \wedge \square_{2} D_{2}\right)$ is true in that world in the original model. Namely, in the case all three children are dirty, children 1 and 2 will know they are dirty after three updates. (2) In both abstractions, $\left\ulcorner[D][K] \square_{1} D_{1}\right\urcorner_{f, g}$ is true at the worlds that correspond to the world which makes $D_{1}$ and $D_{3}$ true. Namely in the case children 1 and 3 are dirty, child 1 will know he is dirty after 2 updates. (3) $\left\ulcorner[D] \square_{3} D_{3}\right\urcorner f, g$ is true at the worlds with only $D_{3}$ true. Namely when only child 3 is dirty, he will know after the first announcement. For the generalization to the $n$ children case, similar abstractions can be made.

Note that whereas all relations in the concrete model are equivalence relations $(S 5)$, this is no longer the case for the abstractions: in the middle abstraction, the must relations can be seen to be non-symmetric, and in the right abstraction, the relation labelled $A$ is no longer transitive (in general the union of two equivalence relations is not necessarily transitive). In terms of the axiom set $S 5$ : some of the axioms are unknown rather than true in the non- $S 5$ abstractions of this example.

\subsection{Encoded Broadcast}

Consider the following simple situation: a television sender wants to broadcast its programs (i.e., streams of bits) only to paying viewers. Therefore, it encodes the stream with a boolean function, let us consider negation. The encoding function has been shared to the registered clients, indexed $1 \ldots n$, while some other unregistered parties, indexed $n+1 \ldots n+m$, do not know it and it should be the case that they do not get access to the programs. A model of this situation can be seen in Figure 3 (up). $b_{1} \ldots b_{n+m}$ are the bits located at the sites of the $n+m$ viewers, currently waiting to be set to the value of the next bit in the stream. The broadcast, to both registered and unregistered users, will consist of one bit $c$, which is the encoding of the actual next bit. In the actual world 


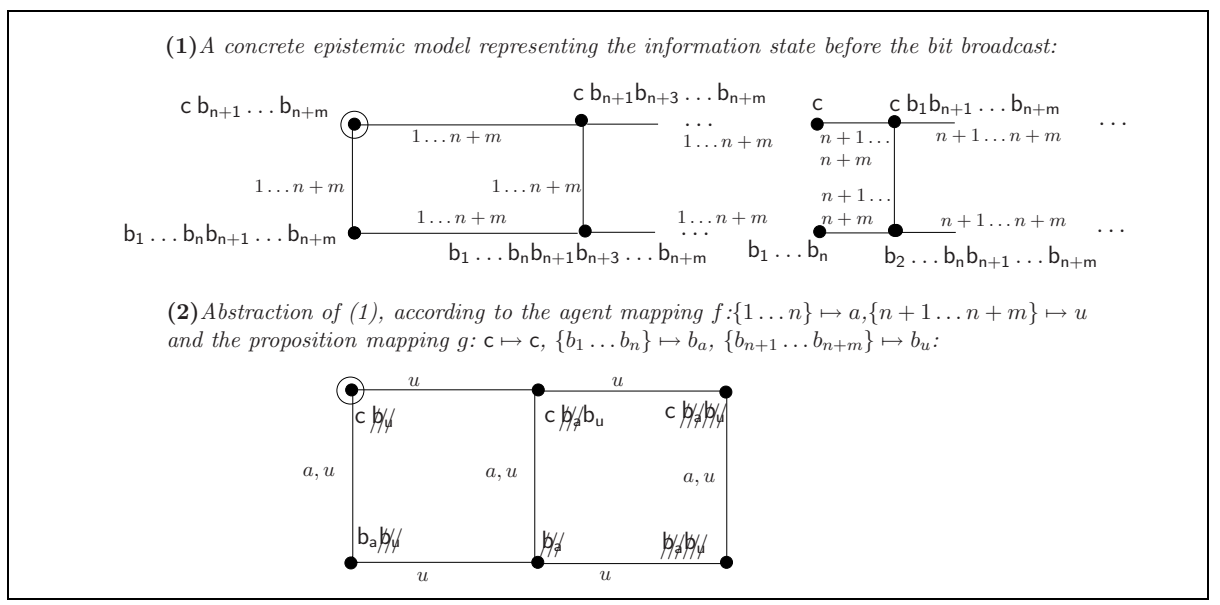

Fig. 3. Epistemic modelling of encoded broadcasting. To keep a clear overview, not all arrows were drawn; the transitive and reflexive closure of the arrow relation forms the intended equivalence. (up): on each row, the first dots stand for a continuation of the sequence of indistinguishable worlds where the valuations range through all the subsets of $\left\{b_{n+1} \ldots b_{n+m}\right\}$. The second dots stand for sequences of worlds where the valuations range through all the subsets of $\left\{b_{1} \ldots b_{n+m}\right\}$ with at least one positive (on top) or negative (on bottom) $b_{i}$, with $i \in\{1 \ldots n\}$. In the possible worlds on the top row, $\mathrm{c}=$ true and on the bottom row, $\mathrm{c}=$ false. The registerd users know that the encoding algorithm ensures $\bigwedge_{i \in\{1 \ldots n\}} \mathrm{c} \leftrightarrow \neg b_{i}$, therefore their indistinguishability relations do not reach worlds where this formula is false. The unregisterd users are not able to distinguish between any two possible valuations. (down): $b_{a}$ and $b_{u}$ can be seen as the receiving bits of a symbolic registered user $a$ and a symbolic unregistered user $u$, respectively. The abstraction in (2) is obtained by mapping all concrete states where $c$ is true and $b_{1} \ldots b_{n}$ are false to the abstract state $c \not k /$, all other concrete states where $c$ is true and $b_{n+1} \ldots b_{n+m}$ are true to $c / p / b_{u}$, and the rest of the concrete states where $\mathrm{c}$ is true to the abstract state $c / p / \phi / \phi$ (a similar mapping for states with $\mathrm{c}$ is false).

(marked with a circle), let us assume that the next bit in the stream is false and hence its encoding is $\mathrm{c}=$ true. We are interested in checking that, after a bit has been broadcasted, (only) the authorized users have received it correctly.

The size of the epistemic model varies obviously with $m$ and $n$ and can be huge, but it is also very regular. The uncertainty relation for every unauthorized agent $i \in\{n+1 \ldots n+m\}$ is the complete graph. Intuitively, this is because such an agent does not hold any information on the encoding function or on any of the waiting bits $b_{1} \ldots b_{n+m}$, so it considers all valuations as possible. An abstraction of this concrete model can be seen in Figure 3 (down). Broadcasting the encoded bit c can be simply modelled by the public announcement of c. The abstract version of this announcement $\ulcorner\mathrm{c}\urcorner f, g$ is still $\mathrm{c}$.

The correct receive property by authorized viewers might be formalized as: $\bigwedge_{i \in\{1 \ldots n\}}[\mathrm{c}] \square_{i} \neg \mathrm{b}_{\mathrm{i}}$ (since the transmitted bit was false). Its translation to the 
abstract context is $[\mathrm{c}] \square_{a} \neg \mathrm{b}_{\mathrm{a}}$, which is true on model (2) in Figure 3. Therefore, according to Theorem 1, all original formulas are true.

The other desired property is that unauthorized users will not receive the intended bit, that is $\bigwedge_{i \in\{n+1 \ldots n+m\}}[\mathrm{c}] \neg \square_{i}\left(\neg \mathrm{b}_{\mathrm{i}}\right)$. The translation of this formula, $[\mathrm{c}] \neg \square_{u} \neg \mathrm{b}_{\mathrm{u}}$ can also be evaluated to true on model (2), meaning, again via Theorem 1. that the value of b doesn't leak to the unauthorized agents. Note that must relations are needed in order to establish satisfiability of such negative knowledge properties. An interesting observation is that, due to the enormous density of arrows in an epistemic model, must relations will occur often enough in abstracted models. This is quite different than the case of LTSs, where most relations in abstracted models are of the may type.

\section{Conclusion}

We proposed a refinement/abstraction framework for KMLTSs, which allows reasoning on small coarse abstract models and transfer the results on refined detailed models. In particular, if the concrete Kripke models are epistemic models, interesting knowledge properties are preserved by refinements and abstractions as shown by two examples.

The theoretical novelty of this work is the extension of traditional abstraction techniques to both the label and proposition mapping, and to a logic containing a dynamic public announcement modality. Both features are of fundamental importance in (epistemic) modelling and verification, which is the main motivation of our work. In order to incorporate the full power of dynamic epistemic modelling, more research is needed on integrating general update constructions as formalized by action models 1 . The abstraction of action models is also practically interesting, as it is shown in [6] that they can be of huge size when modelling protocols. Another goal is to adapt this framework to Interpreted Systems 819], which combines both epistemic and temporal characteristics.

On a practical side, our framework opens the way to automatic epistemic verification of large or even infinite models. Future research should be dedicated to practical problems like generating abstract models directly from textual or formal, but compact, protocol specifications. A possible starting point is the process algebra language of [5].

Acknowledgement. We thank the anonymous referees for their detailed comments. The authors are supported by Dutch NWO project VEMPS (612.000.528).

\section{References}

1. Baltag, A., Moss, L.S.: Logics for epistemic programs. Synthese 139(2), 165-224 (2004)

2. van Benthem, J., van Eijck, J., Kooi, B.: Logics of communication and change. Information and Computation (2006) 
3. Bruns, G., Godefroid, P.: Model checking with multi-valued logics. In: Díaz, J., Karhumäki, J., Lepistö, A., Sannella, D. (eds.) ICALP 2004. LNCS, vol. 3142. Springer, Heidelberg (2004)

4. Burrows, M., Abadi, M., Needham, R.: A logic of authentication. In: Practical Cryptography for Data Internetworks. IEEE Computer Society Press, Los Alamitos (1996)

5. Dechesne, F., Mousavi, M., Orzan, S.M.: Operational and epistemic approaches to protocol analysis: Bridging the gap. In: Dershowitz, N., Voronkov, A. (eds.) LPAR 2007. LNCS (LNAI), vol. 4790. Springer, Heidelberg (2007)

6. Dechesne, F., Wang, Y.: Dynamic epistemic verification of security protocols: framework and case study. In: A Meeting of the minds: Proceedings LORI workshop. Texts in Computer Science, pp. 129-144 (2007)

7. van Eijck, J.: DEMO program and documentation (2005), http://www.cwi.nl/ jve/demo/

8. Fagin, R., Halpern, J.Y., Moses, Y., Vardi, M.Y.: Reasoning About Knowledge. MIT Press, Cambridge (1995)

9. Godefroid, P., Jagadeesan, R.: Automatic abstraction using generalized model checking. In: Brinksma, E., Larsen, K.G. (eds.) CAV 2002. LNCS, vol. 2404, pp. 137-150. Springer, Heidelberg (2002)

10. Halpern, J.Y., O'Neill, K.R.: Anonymity and information hiding in multiagent systems. Journal of Computer Security, 483-514 (2005)

11. van der Hoek, W., Wooldridge, M.: Model checking knowledge and time. In: Bošnački, D., Leue, S. (eds.) SPIN 2002. LNCS, vol. 2318, pp. 95-111. Springer, Heidelberg (2002)

12. Hommersom, A., Meyer, J.-J., de Vink, E.P.: Update semantics of security protocols. Synthese 142, 229-267 (2004); Knowledge, Rationality and Action subseries

13. Hughes, D., Shmatikov, V.: Information hiding, anonymity and privacy: A modular approach. Journal of Computer Security 12(1), 3-36 (2004)

14. Huth, M., Jagadeesan, R., Schmidt, D.: Modal transition systems: A foundation for three-valued program analysis. In: Sands, D. (ed.) ESOP 2001. LNCS, vol. 2028. Springer, Heidelberg (2001)

15. Larsen, K.G.: Modal specifications. In: Automatic Verification Methods for Finite State Systems, pp. 232-246 (1989)

16. Larsen, K.G., Thomsen, B.: A modal process logic. In: Proceedings LICS, pp. 203210 (1988)

17. van der Meyden, R., Su, K.: Symbolic model checking the knowledge of the dining cryptographers. In: Proc. CSFW 2004, pp. 280-291. IEEE, Los Alamitos (2004)

18. Plaza, J.A.: Logics of public communications. In: Proceedings ISMIS 1989, pp. 201-216 (1989)

19. Raimondi, F., Lomuscio, A.: Automatic verification of deontic interpreted systems by model checking via OBDD's. Journal of Applied Logic (2006)

20. van de Pol, J.C., Valero Espada, M.: Modal abstractions in $\mu \mathrm{CRL}^{*}$. In: AMAST, pp. 409-425 (2004)

21. van Ditmarsch, H., van der Hoek, W., Kooi, B.: Dynamnic Epistemic Logic. Synthese Library, vol. 337. Springer, Heidelberg (2008)

22. van Eijck, J., Orzan, S.M.: Epistemic verification of anonymity. ENTCS, 168 (2007) 